This item was submitted to Loughborough's Research Repository by the author.

Items in Figshare are protected by copyright, with all rights reserved, unless otherwise indicated.

\title{
Experimental and computational study of the flow around a stationary and rotating isolated wheel and the influence of a moving ground plane
}

PLEASE CITE THE PUBLISHED VERSION

https://doi.org/10.4271/2019-01-0647

\section{PUBLISHER}

(C) SAE International

\section{VERSION}

AM (Accepted Manuscript)

\section{PUBLISHER STATEMENT}

This paper was accepted for publication in the journal SAE Technical Paper and the definitive published version is available at https://doi.org/10.4271/2019-01-0647

\section{LICENCE}

CC BY-NC-ND 4.0

\section{REPOSITORY RECORD}

Rajaratnam, Eleanor, and Duncan Walker. 2019. "Experimental and Computational Study of the Flow Around a Stationary and Rotating Isolated Wheel and the Influence of a Moving Ground Plane". figshare. https://hdl.handle.net/2134/37496. 


\section{Experimental and Computational Study of the Flow around a Stationary and Rotating Isolated Wheel and the Influence of a Moving Ground Plane Author, co-author (Do NOT enter this information. It will be pulled from participant tab in MyTechZone)

\author{
Affiliation (Do NOT enter this information. It will be pulled from participant tab in MyTechZone)
}

\begin{abstract}
This study investigates the aerodynamic behavior of the flow around a rotating and stationary $60 \%$ scale isolated wheel, with and without the use of a moving ground plane. The aim of this research was to improve the understanding of the fundamental aerodynamic flow features around a wheel and to examine how rotation and moving ground planes modify these and affect the production of drag. A bespoke rotating wheel rig was designed and wind tunnel tests were performed over a range of pre to post critical Reynolds numbers. Force coefficients were obtained using balance measurements and flow field data were obtained using Particle Image Velocimetry (PIV). The unsteady flow field data generated was used to validate unsteady CFD predictions. These were performed using STAR$\mathrm{CCM}+$ and a k- $\omega$ SST Improved Delayed Detached Eddy Simulation (IDDES) turbulence model. This was seen to outperform other models by capturing an increased amount of finer detailed, high frequency vortical structures. The CFD showed good agreement with the experimental results providing, for the first time, a validated numerical methodology. Comparing stationary and rotating wheels the CFD and experimental data both illustrated large scale structural differences in the surrounding flow due to changes in separation and wake structure. The rotating model also exhibited a lower drag at post critical Reynolds numbers, which is corroborated by existing literature. Importantly, the CFD showed minimal difference between a stationary and moving ground plane simulation with a rotating wheel. This is evidence that, provided the wheel is rotating, valid experiments can be performed without the complexity of a moving ground plane.
\end{abstract}

\section{Introduction}

The economic and environmental impact of the car industry drives the need to reduce fuel burn and emissions. One method of achieving this is to reduce aerodynamic drag and as such much research has been focused on improving ground vehicle aerodynamics. To simplify the investigation of aerodynamics and drag reduction a typical vehicle can be considered as a bluff body which generates various regions of separation and wake flow. These flow phenomena are the main factors contributing to the formation of drag. Consequently, a deeper understanding of the influence of various geometric features on the generation of flow separation is crucial. Much of the previous research has focused on separation and wakes generated by the rear end aerodynamics because this accounts for between $40 \%-60 \%$ of the total vehicle drag. However, less focus has been given to wheel and wheelhouse flows and how these interact with the mainstream flow. Ultimately, this flow precedes the rear end of the vehicle and its development will therefore also strongly influence the rear end drag. Hence, this paper focuses on improved understanding of wheel aerodynamics with the aim of validating a numerical methodology.

Traditionally, the primary method in assessing drag has been via balance measurements in wind tunnels. However, analyzing the associated flow phenomena is challenging due to the difficulty in obtaining accurate flow field measurements. Furthermore, simulating wheel rotation and the relative motion with the road leads to complex and expensive test facilities. Hoerner [1] states that the ideal reproduction of any road surface involves a belt moving under the model at the same speed as the freestream flow. These are in use widely but there is still limited high quality published experimental research examining drag reduction techniques with respect to wheel aerodynamics. With the improving accuracy of CFD and the ease with which geometry modifications, wheel rotation and relative motion can be accommodated numerical simulations have become an integral part of evaluating, understand and developing vehicle aerodynamics.

\section{Existing Literature}

Morelli [2], Stapleford \& Carr [3] and Cogotti [4] were some of the first to investigate an isolated wheel close to the ground, and the importance of rotation and contact between the tire and moving ground plane. Due to practical limitations, these preliminary studies of isolated wheels were usually performed without the use of a moving ground plane or the ability to accurately obtain force measurements. Morelli [2], who tested both a stationary and rotating isolated wheel configuration with a stationary ground plane, found that the gap between the wheel and ground, a necessary requirement for the motor to drive the wheel, caused a change in the flow field and created a negative time-averaged lift. This was due to a Venturi effect which generated an artificially low pressure under the wheel. Stapleford \& Carr [3] and Cogotti [4] tried to rectify this by sealing the gap with either strips of paper or foam rubber. Stapleford \& Carr [3] found that this, in tandem with a stationary ground plane and a rotating wheel, generated a positive lift which contradicted the results of Morelli [2].

The importance of the rotation of wheels and the consequential effect on aerodynamic behavior has been studied in depth since the aforementioned studies. Fackrell [5] and Fackrell \& Harvey [6] published data which is still currently used in experimental validation 
of numerical modelling. Their work was the first to experimentally study a rotating wheel in direct contact with a moving ground plane. This was succeeded by work completed by Mercker et al. [7, 8] who used a more advanced belt technique allowing for simultaneous force component measurements to be carried out. Measurements showed that the wheels and wheel arches for a production Audi A3 fitted with a smooth underbody, accounted for up to $33 \%$ of the aerodynamic drag. Wickern et al. [9] continued this work with Audi and tested a vehicle with wheels and wheel arches using a moving belt facility and the vehicle suspended from a rear-located support. It was found that the inclusion of the wheels represented $25 \%$ of the total drag of the car. These studies outlined the significance of wheel motion to ensure correct wake development and that wheel and ground contact was the only way to estimate true aerodynamic coefficients. These studies highlight the importance of wheel/ground motion but fall short of definitively stating the degree of complexity required to adequately capture the flow field topology and understand the sources of drag. The current paper aims to address this by using a combination of high fidelity experimental and numerical techniques to examine an isolated wheel in contact with the ground both with and without rotation and a moving ground plane.

The findings that both wheel and ground plane interaction has a potentially strong effect on a vehicle's total lift and drag was also shown more recently both experimentally and computationally on full scale vehicles by Elofsson et al. [10], Duncan et al. [11] and Wäschle [12]. Here, it was the authors' main objective to quantify the impact of different elements on the vehicle's global aerodynamic performance to discover whether or not it is necessary to take them into account for future testing or numerical modelling.

Experimentally, a large reduction in $\operatorname{drag}\left(C_{D}=0.01-0.02\right)$ was measured on all vehicle configurations tested with rotating wheels and a moving ground center-belt. The studies concluded that wheels had a major effect on car aerodynamics and therefore the reasons for the development of the associated flow must be correctly understood. Although the main flow features have been studied previously, authors do not agree on the exact flow topology surrounding a rotating wheel. Cogotti [4] proposed the theoretical wake model shown in Figure 1, by defining an isolated wheel as a narrow span cylinder bluff body. Here, a) has no rotation and the diagram represents the wake pattern due only to the shape of the wheel; with two pairs of counter rotating vortices generated off the wheel shoulders. b) With rotation, the diagram shows the pair of vortices created from the wheel sides, but these have not been observed in further studies performed by Mercker et al. [7] and more recently Knowles et al.[13]. c) Illustrates the combination of both a) and b), with the inclusion of the ground effect.
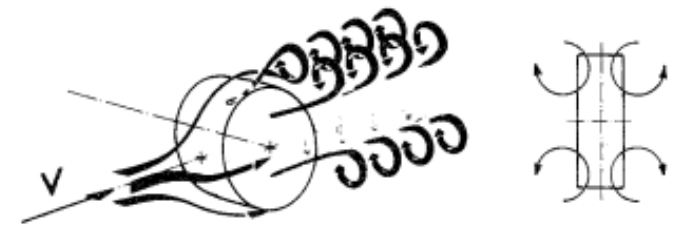

a)
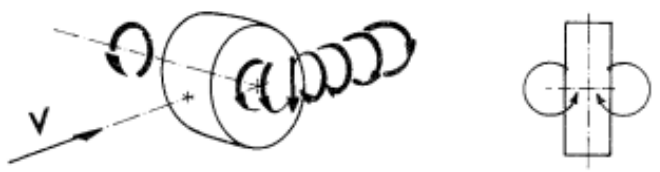

b)
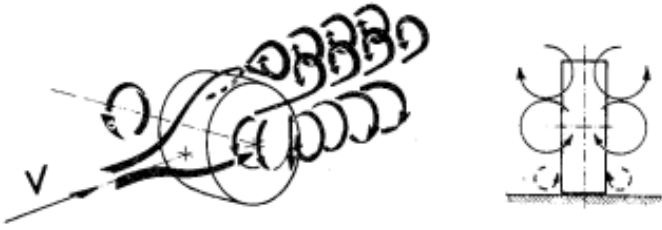

c)
Figure 1. Theoretical Wake Model [4]

This model was validated and improved further by Mercker et al. [7] who presented a schematic of near wake vortical structures including two ground plane jetting vortices as shown in Figure 2. Here, a) displays a stationary wheel. As the wheel is larger in height than width, the flow rate, $\dot{Q}$, is greater at the sides than either the top or bottom. Along the axis of symmetry, oblique flow, $V$, is directed towards the outer edges of the wheel. It was stated that the vorticity, $\Gamma$, of all four vortex structures will be the same. b) Shows also a stationary wheel with a fixed ground. Flow that escaped beneath the wheel at a) is now deflected to the sides increasing the strength of the ground vortices and moving the stagnation point, $S P$, down. This increases drag and lowers lift as base pressure is decreased. A horseshoe vortex, originating from the viscous flow separation at the ground, is generated at the front of the wheel. c) Depicts a rotating wheel. The stagnation point is shown to move upwards due to the Magnus effect exhibited on the wheel, although this is suppressed due to the ground effect, creating downforce. The corresponding downwash reduces the ground effect vortex strength and horse-shoe wake which decreases both lift and drag as base pressure is increased. As less flow is directed towards the upper wheel section, more flow is pushed to the sides, increasing the effect of the upper vortex pair. 

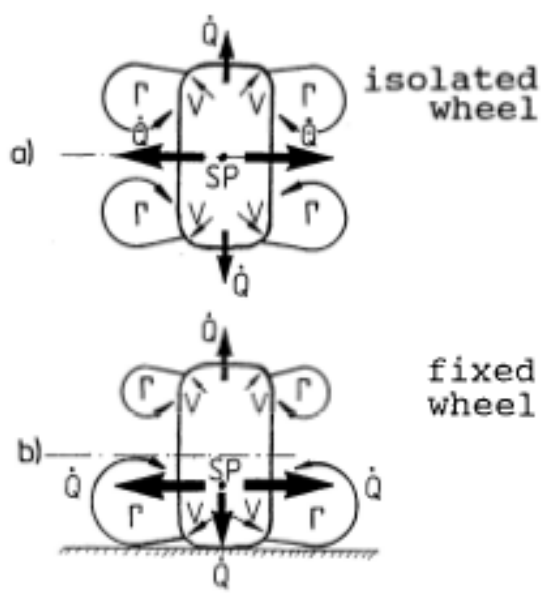

fixed wheel

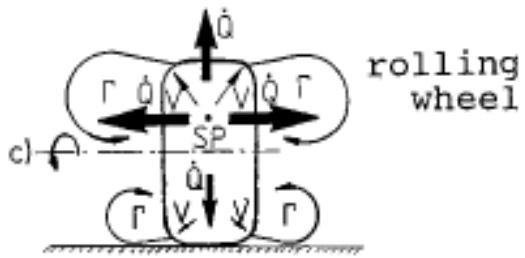

Figure 2. Flow Model for the Vortex Flow around an Isolated, Fixed and Rotating Wheel [7]

Fackrell [5] used surface pressure measurements, wake surveys and smoke visualizations to compare both rotating and stationary wheels. It was found that the flow travelling past the wheel and into the near wake created a pair of counter rotating vortices emanating from the tire shoulders. Surface pressure distribution showed that upstream of the contact area, the rotation of the wheel and moving ground behaved like a 'viscous pump' and caused pressure coefficients to rise above unity $\left(C_{P}>1\right)$. A lateral jet, referred to as the 'Jetting Phenomena', is produced on either side of the wheel creating two vortical structures near the ground as shown in Figure 2. A negative pressure peak downstream of the contact patch was predicted but not shown experimentally until more recent work conducted by Mears et al. [14]. These vortices were stronger for the stationary wheel than for the rotating, once again in agreement with the flow model proposed by Mercker et al. [7]. It was also shown that the rotating wheel produced a taller and narrower wake than that of the stationary due to the boundary layer separation and ground jetting vortices which has been corroborated computationally by Wäschle [12], producing lower drag and lift coefficients than when stationary $\left(C_{D}\right.$ of 0.58 compared to 0.77 ).

Mears et al. [14] revisited some of the main observations of Fackrell [5] using a purely experimental investigation. Particle Image Velocimetry (PIV) was used to capture the flow field behavior around the wheel in conjunction with surface static pressure tappings. The lift and drag coefficients for both rotating and stationary configurations matched well with those of Fackrell [5] with wheel rotation reducing the drag. From the pressure coefficient plots it was observed that, for both rotating and stationary configurations, the leading stagnation point was depressed by approximately $5^{\circ}$ from the horizontal. The rotating experiment also exhibited a higher than unity pressure coefficient upstream of the contact patch in line with
Fackrell's 'Jetting Phenomena', this was captured in early computational studies performed by Axon et al. [15], however the magnitude of the pressure was shown to be highly mesh sensitive.

Although total pressure wake measurements can be unreliable when the angle between the probe and the flow is large (Wäschle et al. [16]), Knowles et al. [13] used this measurement technique to determine the directional characteristics of the vortices induced. It was shown that the inclusion of a support sting suppressed and prevented the development of some of the suggested vortex structures shown in Figure 1. It was also presented that the ground vortex pair is more dominant in strength than the upper pair and that these merge within one diameter downstream of the wheel base. This has also been corroborated computationally by Wäschle [12]. It was further explained that vortex induced flow regions away from the tire profile are due to impingement of the flow at the front of the contact patch which produce the lateral jetting vortex pair that widen the wake of the wheel, also presented computationally by Axerio-Cilies \& Iaccarino [17].

Croner et al. [18] computationally showed that extreme $C_{P}$ was exhibited on either side of the contact patch $\left(C_{P}=-13.5-15.6\right)$ which again gives evidence of Fackrell's 'Jetting Phenomena'. However, it was also shown that these counter-rotating vortices are quasi-symmetric, and that symmetry is lost due to lateral motion as well as model set-up. The wake was not depicted to generate classical Von Karmen vortex shedding as structures upstream of the contact area were shown to be affected by the downstream wake region.

The upper vortex structures in both Figure 1 and Figure 2 are affected by both the stagnation point and the separation over the crown of the wheel. The location of this separation point is a characteristic that is often used for the validation of numerical studies, where inaccurate prediction can lead to wake flow field discrepancies stated by AxerioCilies \& Iaccarino. [17]. Fackrell [5] used smoke visualizations to explain drag differences and show that separation occurred further upstream for the rotating wheel, within the favorable pressure gradient, when compared to the stationary wheel which separated in the adverse gradient regime. The pressure gradient counteracts the impeding effect of the shear stress within the viscous boundary layer and it was postulated that this carries the turbulent fluid upstream, preventing attachment and causing earlier separation. Fackrell \& Harvey [6] showed that by averaging the base pressure, a quasiconstant region of pressure can be found and that the end of this region can be construed as the point of separation, although this point has been shown to be sensitive to geometry parameters such as tire profile [6], computational meshes [19] and turbulence models [20]. Mears et al. [14] defined the point of separation using the $C_{P}$ distribution, however this method has not been validated.

As hypothesized by Fackrell \& Harvey [6] and validated by more recent experimental work by Regert \& Lagos [20] , Croner et al. [18] and via direct numerical simulations by Pirozolli et al. [21], the fluid entrainment at the wheel surface meant that separation did not originate from there, rendering surface pressure analysis inadequate. Instead, it was found to come from the thin layer of fluid surrounding the surface that counteracts the freestream direction, thus another method for finding this separation point involves identifying the saddle point topology. The location of this was shown to differ to that of the pressure suction peak. 
This crown separation is integral to capture and simulate correctly as it has been shown to produce differing wake structures depending on the method employed. For example, McManus \& Zhang [22] used both steady RANS and unsteady uRANS to identify the regions of separated flow with iso-surfaces and pressure coefficient results, showing that the flow separated over the upper surface generating an arch shaped vortex with approximately the same height and width of the wheel. This region was observed to be smaller for the rotating wheel indication again a lower drag being generated. Wäschle[12] however utilized both RANS and VLES (PowerFLOW) to display a ring vortex within this separated flow region. Croner et al. [18] showed with both experimental PIV and uRANS simulations results that an arch shaped structure was generated, corroborating the results of McManus \& Zhang [22].

In summary, there have been many studies on wheel aerodynamics considering isolated wheels both stationary and rotating and in proximity to the ground. Most studies agree that rotation and the presence of the ground can have a significant effect on the flow topology and subsequent drag. In many recent studies it has been assumed that a moving ground plane is needed to generate the correct flow physics. However, insufficient evidence is presented to support this and thus justify the additional cost and complexity of a moving ground plane. Consequently, this paper will address this question directly. Can the defining flow features prevalent in a rotating wheel in contact with the ground be created without the use of a moving ground plane? Specifically, the aims of the work are to:

- Design and implement a wind tunnel test rig that will enable the measurement of drag for an isolated wheel with and without rotation.

- Use particle image velocimetry (PIV) to provide high fidelity experimental data describing the flow field topology at various speeds with and without rotation.

- Develop a validated CFD model, based on the experimental setup.

- Use the CFD model to further analyze the flow and to definitively examine the effects of a moving ground plane and establish if it is a requirement to generate the global flow features of a rotating wheel.

\section{Experimental Methodology}

This study investigates the aerodynamic behavior of a $60 \%$ scale isolated wheel driven by a rotating drum system in a wind tunnel without the use of a moving ground plane, MGP, through a range of pre to post critical Reynolds numbers, with one aim being to identify the differences in flow behavior between a typical moving ground plane configuration and that of which was being tested. The rig was designed, manufactured and tested at Loughborough University where 2D Particle Image Velocimetry, PIV, and balance data was obtained experimentally. All experiments were performed in the Loughborough University Large Wind Tunnel, Figure 3, details of which can be found in [23]. The normal operating velocity of the tunnel is $40 \mathrm{~m} / \mathrm{s}$ with a freestream turbulence of approximately $0.2 \%$. The design considerations of the experimental model were based on tests conducted by Cogotti [4] that were performed over a range of Reynolds numbers for a stationary wheel at the Pininfarina Wind Tunnel. A trend was found that showed an obvious transition for both lift and drag from pre (sub) to post (super) critical flow behavior, similar to what was found by Hoerner [1]. It was postulated that for a full-size stationary wheel, Reynolds numbers of over $1 \times 10^{\wedge} 6$ (post critical) were needed if one was to obtain accurate and reliable post critical drag separation. However, for this work, practical limitations, such as wind tunnel size and blockage, meant that it was not possible to test at full scale, therefore it was decided that a minimum of a $60 \%$ scale model would be utilized. The final design, as illustrated in Figure 4, and shown with the wind tunnel floor in Figure 5, comprises of a wheel with a width of $140 \mathrm{~mm}$ and a diameter $(\phi)$ of $350 \mathrm{~mm}$, which in turn if tested at $30 \mathrm{~m} / \mathrm{s}$ gives a Reynolds number based on the diameter of approximately $7.2 \times 10^{5}$. It was found that this velocity lay comfortably within the post critical region ensuring separation was accurately captured. 3D printed inserts, colored pink and displayed in Figure 5, were also manufactured to greatly reduce the gaps around the contact patch between the wheel and the drum, minimizing suction effects through the floor.

The $60 \%$ diameter value is based on a range of saloon and SUV average wheel diameters, where these can vary between $600 \mathrm{~mm}$ $750 \mathrm{~mm}$. A motor is fixed to the bottom plate and used to power the $\operatorname{drum}(\phi=200 \mathrm{~mm})$ rotation which in turn drives the main wheel. A $1: 1$ drive ratio was used for the connecting pulley and belt system. The mounting frame is made from aluminum as well as the drum of the wheel and roller, whilst the end caps of both are made from nylon. This allows for freedom in the styling of the hubs, providing the potential of creating spokes to simulate a porous flow through the wheel at a later stage.

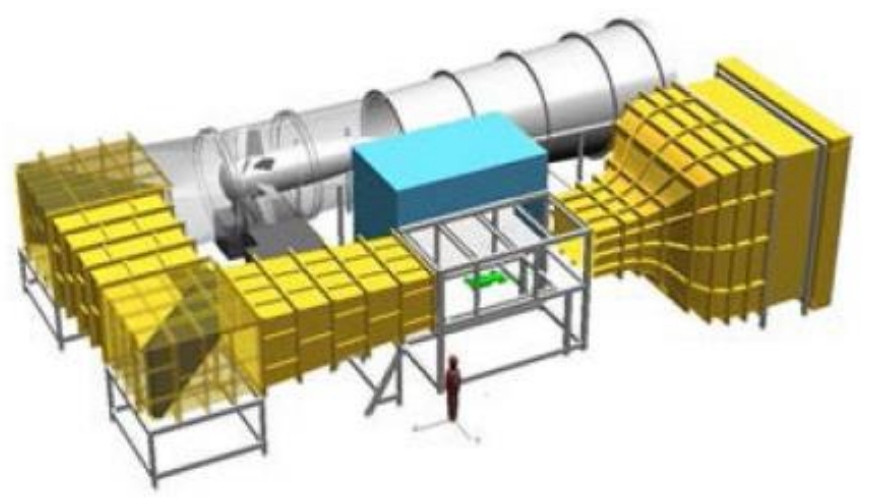

Figure 3. Loughborough University Wind Tunnel [23] 


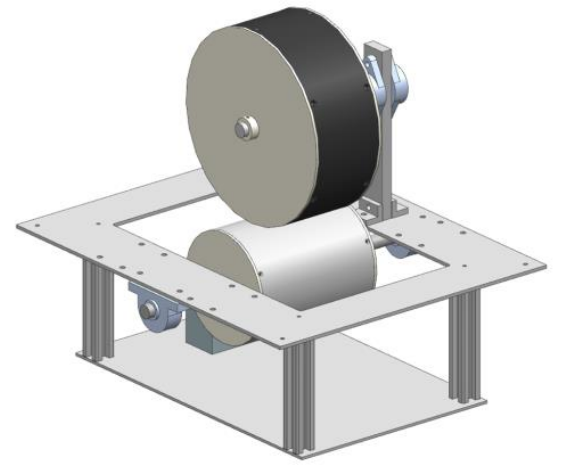

Figure 4. Isolated Wheel Rig Design

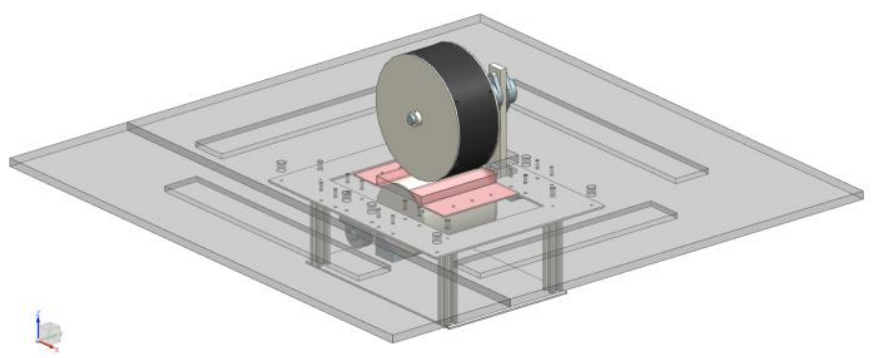

Figure 5. Isolated Wheel Rig: Location of Wind Tunnel Floor

\section{Balance Measurements}

Balance data was sampled with a six-component balance for 300s at a sampling rate of $300 \mathrm{~Hz}$ which generates 90000 data points per test. Prior to testing, repeatability checks were performed where the model was set up in the tunnel more than once and the yaw sweep results compared. The balance measurements are repeatable to $\Delta C_{D}=0.008$ when taken within the same test period. For this study, a typical drag coefficient $C_{D}=1.06$ was found for a stationary wheel configuration at $30 \mathrm{~m} / \mathrm{s}$ and $0^{\circ}$ yaw.

Due to difficulty in achieving full isolation of the balance, balance measurements were only taken for the stationary wheel configurations.

The wind speeds and thus Reynolds numbers have been corrected for blockage using the standard continuity based MIRA blockage correction [24]:

$$
U_{\text {corrected }}=U_{\text {measured }} \cdot \frac{100}{100-\text { Blockage }}
$$

Where,

$$
\text { Blockage }=\frac{\text { Model frontal area }}{\text { Tunnel working section area }} \cdot 100
$$

\section{PIV Measurements}

Two 5.5 Megapixel dual-frame LaVision Imager sCMOS cameras with 16 bit $2560 \times 2160$ pixel CCD sensors were used to obtain the PIV images. The light sheet was generated using a dual pulse $532 \mathrm{~nm}$ $200 \mathrm{~mJ}$ Litron laser that generates a sheet approximately $1 \mathrm{~mm}$ thick across the measurement plane. The flow was seeded in both the tunnel contraction and through the floor at the start of the working section with DEHS, generating particles with a mean diameter of less than $1 \mu \mathrm{m}$.

Data was obtained and processed using Davis software. The calculation of the vectors is reliant on defining the average particle displacement in an interrogation cell by calculating the spatial correlation between the first and second frame of a raw image pair. Geometric masking and a red filter within the tunnel was used to reduce problems of glare and laser reflection. A multi-pass processing approach was employed, starting with a $128 \times 128$ pixel interrogation cell, which is used to calculate a reference vector field, reducing in size to $24 \times 24$. Interrogation cells were overlapped by $50 \%$ to improve data yield and thus the validation process [25].

Figure 13 displays the location of the PIV measurement planes obtained for this study. All time averaged data have been calculated from 1000 instantaneous vector fields at a sampling rate of $5 \mathrm{~Hz}$.

\section{Numerical Methodology}

A Navier Stokes based CFD solver, STAR-CCM+ is an all-inclusive package, containing a processor, solver and post-processor. For all simulations, the cross-sectional area of the domain was set to match dimensions of the Loughborough University Scale Wind Tunnel, as shown in Figure 3. The Trimmed Cell mesher as well as a set of volumetric controls, were applied within the domain to control the cell size for each refinement zone, as shown in Figure 6. Trimmed (hexahedral) mesh cells are known to be beneficial due to the minimal cell skewness generated and these generally excel at solving external flow behavior [26]. Prism Layers, as illustrated around the wheel in Figure 7, were also employed to capture the boundary layer with a $\mathrm{Y}^{+}<1.5$ set on all relevant surfaces, signifying the viscous sublayer is resolved. As $\mathrm{Y}^{+}$is dependent on velocity and correct simulation of the boundary layer flow is integral to this piece of work due to the difficulty in accurate prediction of the separation point over the top of the wheel, the prism layer mesh and thus $\mathrm{Y}^{+}$was altered accordingly for each Reynolds number case tested so that a $\mathrm{Y}^{+}<1.5$ was maintained throughout.

DES is a hybrid modelling approach that uses RANS equations to model the near wall flow whilst LES, unsteady, time dependent equations, is utilized in the far-field. These unsteady equations are best utilized in particular transient cases where the unsteadiness is 
either imposed or inherent, usually defined by high resolution vortex shedding that induces large sections of separated flow [26]. However, care has to be taken when configuring the correct solver settings as simulations require specific temporal discretization as time scales of the turbulence need to differ from the mean flow unsteadiness for the simulations to predict reliable and accurate results [26]. DES models are arranged so that boundary layer and conservative vector flows are primarily solved using a RANS closure model, however if the grid created is of a fine enough density it will be solved using a LES subgrid scale model in detached flow regions.

A K-Omega SST Improved Delayed Detached Eddy Simulation, IDDES, STAR-CCM+ turbulence model was used and found to outperform other computational models such as URANS by capturing an increased amount of finer detailed, high frequency vortical structures whilst also showing a good agreement with experimental results found by Mears et al. [14]. This will be discussed further in the following results section. A time step of $2.5 \times 10^{-5} \mathrm{~s}$ was used for all DES simulations as it ensured satisfactory temporal discretization. Due to added complexity, the drum rotating system was not modelled computationally, therefore the wheel was located directly on top of the ground plane. The rotating wheel was modelled with a moving reference frame as a translational boundary condition with respect to a cylindrical coordinate system that was located at the center of the axle rod.

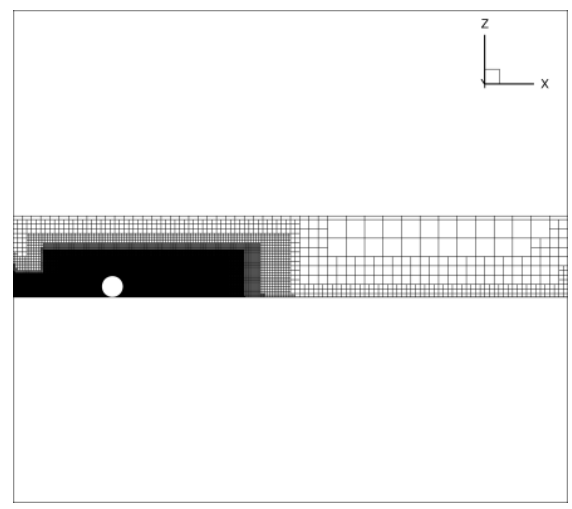

Figure 6. Computational Mesh: Full Domain

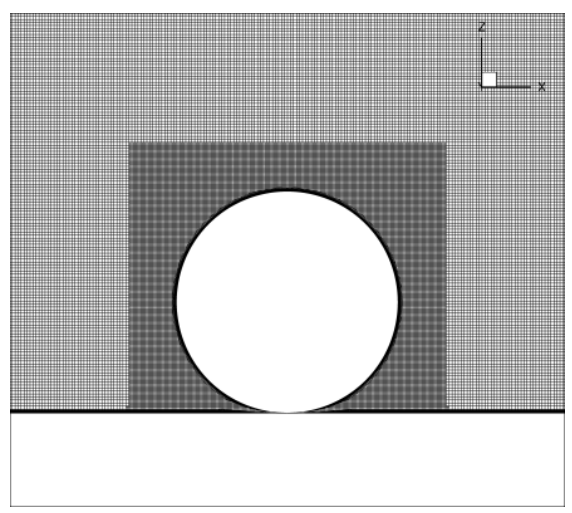

Figure 7. Computational Mesh: Wheel Detail 


\section{Results}

\section{Preliminary Validation - Surface Pressures}

Figure 8 illustrates the angular notation for all analyses presented in this paper regarding both stationary and rotating wheels. This is valid for all PIV results and computational comparisons.

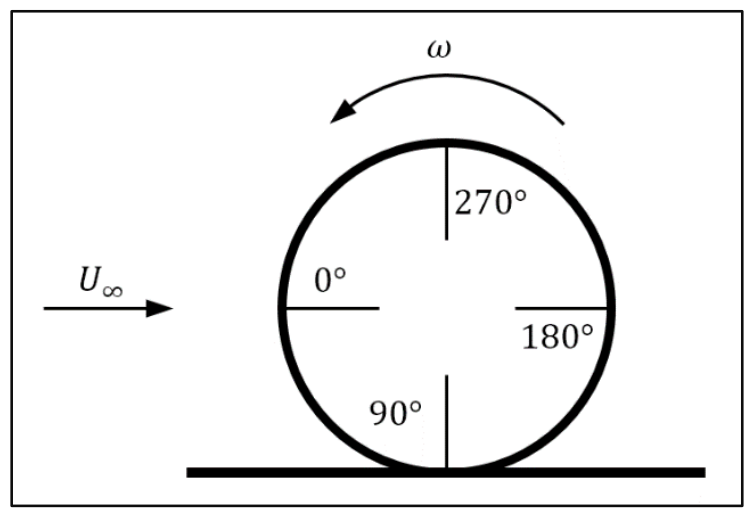

Figure 8. Angular Notation for Wheel

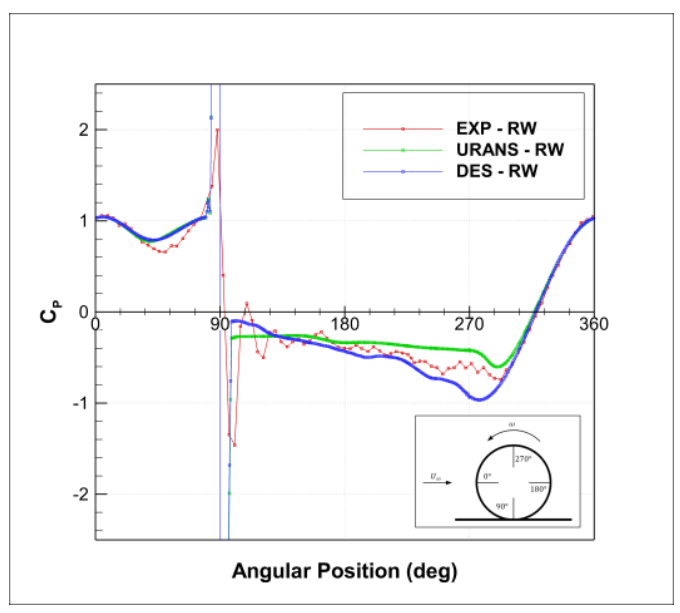

Figure 10. Rotating Wheel: Experimental vs uRANS and DES Centreline Mean $\mathrm{C}_{\mathrm{P}}$

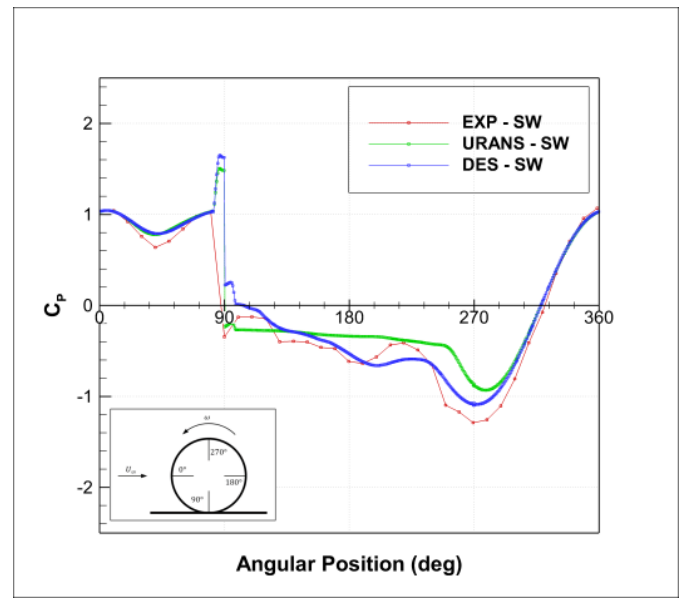

Figure 9. Stationary Wheel: Experimental vs uRANS and DES Centreline Mean $\mathrm{C}_{\mathrm{P}}$

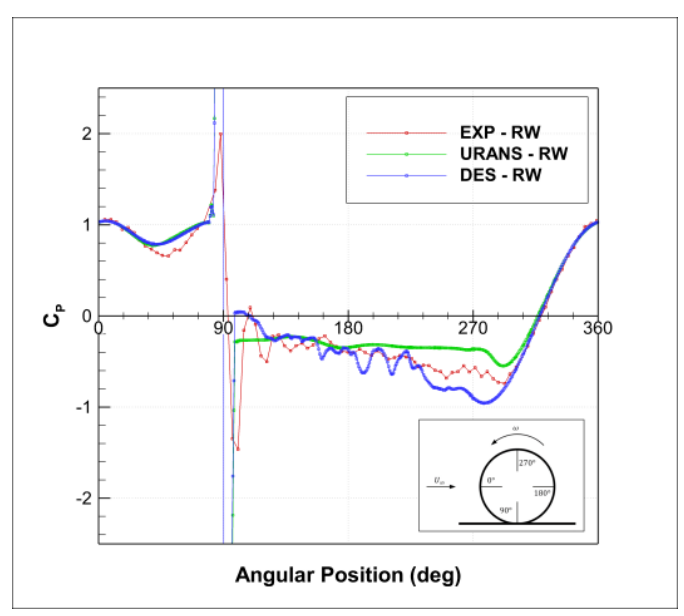

Figure 11. Rotating Wheel: Experimental vs uRANS and DES Centreline Instantaneous $\mathrm{C}_{\mathrm{P}}$ 
The coefficient of pressure has been defined by the following equation:

$$
C_{P}=\frac{p-p_{\infty}}{\frac{1}{2} \rho_{\infty} V_{\infty}^{2}}
$$

Where,

$$
\begin{aligned}
& p=\text { Static Pressure at Evaluation Point } \\
& p_{\infty}=\text { Static Pressure in the Freestream } \\
& \rho_{\infty}=\text { Density in the Freestream } \\
& V_{\infty}=\text { Velocity in the Freestream }
\end{aligned}
$$

Preliminary assessment of the CFD methodology was made by comparing the predicted surface pressure coefficients to experimental data from Mears et al. [14]. The numerical data presented has been averaged over $0.5 \mathrm{~s}$ after a settling period of $0.5 \mathrm{~s}$, generating a mean coefficient of pressure field. Figure 9 and Figure 10 compare the uRANS and DES predictions, both of which have the same computational mesh, to the experimental data for a stationary and rotating wheel respectively. Both CFD models broadly capture the pressure distribution but the DES better predicts the detail whereas the uRANS over-smooths the results; this is especially noticeable for the stationary configuration. The DES results presented in Figure 11, which displays the pressure data at an instantaneous time of $1 \mathrm{~s}$, provides evidence that iteratively there is far more variation in the pressure distribution, a result typical of a highly unsteady rotational flow, however the uRANS shows minimal difference between averaged and instantaneous data. This is due to the existing statistical averaging term within the uRANS solver which still only models the Navier Stokes equations at every time step.

The stagnation point just before the contact patch $\left(\theta=90^{\circ}\right)$, was stated for the stationary wheel as a characteristic caused by the contact with the ground plane [14]. For the rotating wheel however, extra energy is injected into the flow and the increase in magnitude is caused by the viscous interaction of the two boundaries (wheel and ground plane), that converge and squeeze the air to a local static pressure coefficient in excess of unity.

Both models are successful in predicting a pressure coefficient greater than one immediately upstream of the contact patch $\left(\theta=90^{\circ}\right)$ and a large reduction immediately downstream for the rotating wheel. This is attributed to 'Fackrell's Jetting Phenomena' [5] and is consistent with the observations in several other computational studies such as McManus and Zhang [22] and Axon et al. [15]. The magnitude of the maxima and minima apear overpredicted for both stationary and rotating wheel but this is more likely due to a lack of resolution in the experimental pressure taps compared to the computational grid. The rotating peaks are also similar in magnitude and therefore cancel each other out, having a negligible effect on the model's lift. Although the strong negative pressure over the top of the wheel, found especially in the stationary configuration $\left(225^{\circ}<\theta<\right.$ $\left.315^{\circ}\right)$, contributes towards the higher lift force found $\left(C_{L}=0.672\right)$ than when compared to the rotating wheel $\left(\mathrm{C}_{\mathrm{L}}=0.617\right)$.

Using static pressure readings to indicate the separaton point can be quite unreliable [18], however this was the method employed by Mears et al. [14] for this research and previously by Fackrell [5] who defined it as the point at which pressure recovery ceased. Using this evaluation technique, the comparison of the separation points and the approximation of the quasi-constant base pressure can be found in Table 1.

Table 1. Comparison of Separation Points and Base $C_{P}$

\begin{tabular}{|c|c|c|c|c|}
\hline \multirow{2}{*}{ Method } & \multicolumn{2}{|c|}{ Separation Point $\left(^{\circ}\right)$} & \multicolumn{2}{|c|}{$\begin{array}{c}\text { Quasi-Constant Base } \\
\text { C }_{\mathbf{P}}\end{array}$} \\
\cline { 2 - 5 } & $\begin{array}{c}\text { Stationary } \\
\text { Wheel }\end{array}$ & $\begin{array}{c}\text { Rotating } \\
\text { Wheel }\end{array}$ & $\begin{array}{c}\text { Stationary } \\
\text { Wheel }\end{array}$ & $\begin{array}{c}\text { Rotating } \\
\text { Wheel }\end{array}$ \\
\hline $\begin{array}{c}\text { Experiment } \\
\text { (Mears et al. } \\
[14])\end{array}$ & 230 & 290 & -0.6 & -0.4 \\
\hline URANS & 250 & 280 & -0.4 & -0.4 \\
\hline DES & 240 & 270 & -0.5 & -0.5 \\
\hline
\end{tabular}

The data in Figure 9 illustrates the decreasing pressure coefficient during the quasi steady region and that the CFD (DES) agrees well with the general trend as well as the location if not the absolute value of the suction peak. With rotation, Figure 10, for the experimental data, Mears et al. [14] states separation moves to $290^{\circ}$. The CFD also shows an upstream movement of the minima from stationary to rotating but only by around $30^{\circ}$ compared to the $60^{\circ}$ seen in the experiment. Overall, the base pressures are also better matched in magnitude and graphical trend with the DES rather than the uRANS predictions. The main difference in the DES results is shown in the prediction of the suction peak around the hypothesized point of separation, this is more noticeable for the rotating wheel. However this can attributed to what was shown by Mears et al. [14] who found when comparing experimental data with Fackrell's, pressure distribution differences can be attributed to the different edge profile geometry and aspect ratio of the wheel having an effect on the flow field as well as Reynolds number used. In the cases simulated, the model used did not have the rounded edges of a tire and the Reynolds number was doubled $(14 \mathrm{~m} / \mathrm{s}$ to $30 \mathrm{~m} / \mathrm{s})$. These changes would directly affect the strength of the vortex structures produced such as the upper rear counter rotating pair off the wheel shoulders which controls both the separation point and the strength of the suction peak located here.

The data clearly shows that the DES simulations better match the measured variations in $C_{P}$ despite some small differences in the absolute values. Hence the DES model was used for all future simulations reported herein. 


\section{Lift and Drag Data}

\section{Stationary Wheel}

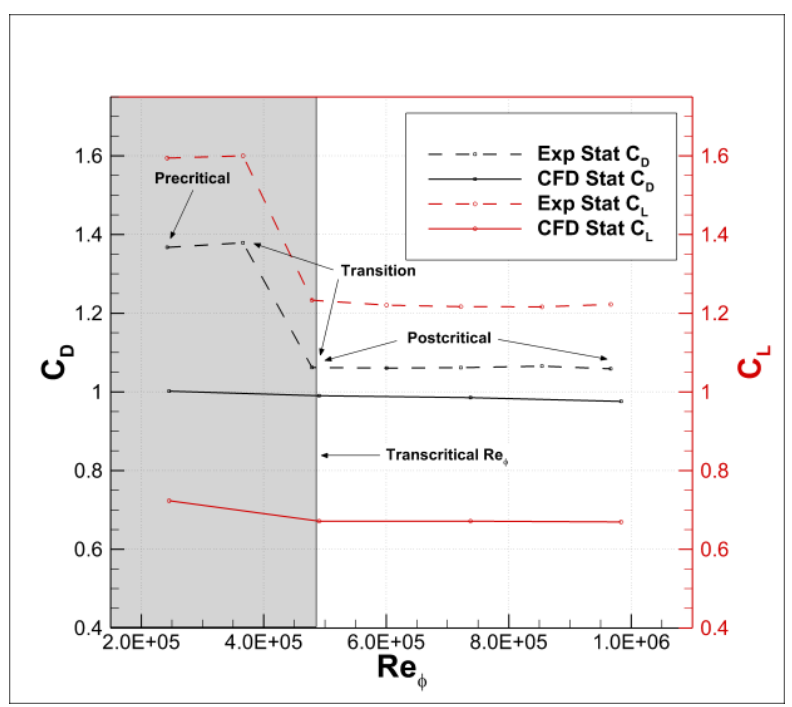

Figure 12. Stationary Wheel: Computational and Experimental Comparison of $\mathrm{C}_{\mathrm{D}}$ and $\mathrm{C}_{\mathrm{L}}$ against Reynolds Number

The Reynolds Number $(\phi)$ has been defined with the following equation:

$$
R e=\frac{\rho v \phi}{\mu}
$$

Where,

$$
\begin{aligned}
& \rho=\text { Density of Fluid Air }=1.225 \mathrm{kgm}^{-3} \\
& v=\text { Velocity of Air } \\
& \phi=\text { Diameter of Wheel }=0.35 \mathrm{~m} \\
& \mu=\text { Dynamic Viscosity of Air }=1.789 \times 10^{-5} \mathrm{kgm}^{-1} \mathrm{~s}^{-1}
\end{aligned}
$$

The experimental data shown in Figure 12 highlights the pre to postcritical flow regimes where the lift and drag coefficients drop rapidly as the increased Reynolds number promotes boundary layer transition. Clearly this will not be captured by the CFD as the model is fully turbulent. However, beyond the critical value the predicted lift and drag coefficients become independent of Reynolds number. The transcritical Reynolds number $\left(\sim 5 \times 10^{5}\right)$ corresponds to a tunnel speed of $\sim 20 \mathrm{~m} / \mathrm{s}$ or full-scale speed of close to $40 \mathrm{kph}$. This is much lower than a typical cruise speed. Hence, all further tests were conducted at a tunnel speed of $30 \mathrm{~m} / \mathrm{s}$ (full-scale $\sim 60 \mathrm{kph}$ ) which, at a Reynolds number close to $7.2 \times 10^{5}$ avoids transitional effects. Experimental balance data was taken for the stationary configuration only due to small vibrations affecting the highly sensitive balance when the wheel was rotating.

Comparing the measured and predicted data, Figure 12 shows that the CFD consistently under-predicts both the lift and drag coefficients. It is most evident for the lift which is under predicted by approximately $50 \%$ whereas the drag is closer to $10 \%$. This level of discrepancy can be attributed to: (i) due to difficulties in meshing the contact patch between the wheel and the floor-mounted drum used to drive the rotation, it is omitted from the numerical model and, (ii) possible leakage in this region. For example, flow leakage via the gap can reduce the pressure close to the contact patch, creating a larger pressure difference top-to-bottom equating to a higher overall lift. This has been observed experimentally by Stapleford and Carr [3], Cogotti [4] and Mears et al. [14] and numerically by Wäschle et al. [16], and Croner et al. [18].

\section{PIV Measurements}

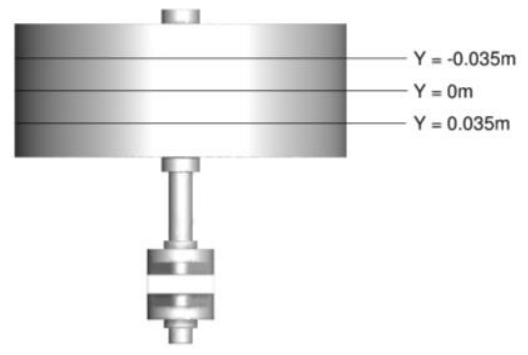

Figure 13. PIV Measurement Plane Locations

Figure 13 illustrates the three different vertical planes that PIV data was obtained for. However only the vertical centerline $(Y=0)$ plots will be used for the comparison with computational data in this paper.

\section{Discussion}

It has been claimed in existing literature that the differing rear wakes created by the varying pressure and flow velocity distributions over the wheel is the main reason a smaller magnitude of drag force is produced by the rotating wheel. The stationary wheels shown in, Figure 14, Figure 16 and Figure 20, colored by respectively $\frac{U_{x}}{U_{\infty}}$ and $\frac{U}{U_{\infty}}$, portrays a wake which is marginally shorter in height but wider in width, dissipating at a slower rate than that of the rotating wheel that feeds energy into the wake, reducing the losses [18]. These factors explain the $\mathrm{C}_{D}$ difference between the stationary wheel (CFD: $\mathrm{C}_{\mathrm{D}}=$ $0.988)$ and the rotating wheel $\left(C F D: C_{D}=0.985\right)$.

The two pairs of counter rotating vortices coming off the wheel shoulders and floor, shown more clearly in Figure 24 by structure pairs A (shoulders) and B (floor), creates a slightly larger wake for the stationary wheel, although the velocity magnitudes found within the wake for the rotating wheel are slightly lower. The reason that the drag recorded for the rotating simulation is not further reduced, as expected from the literature, is likely due to the substantial interfering effects of the stand and flange bearing location. These factors can be used to explain the higher drag force $\left(C_{D}=1.024\right)$ found for the moving ground plane simulation.

The main difference in flow topology is the separation bubble that occurs over the first quadrant of all rotating cases. This was discussed in the pressure coefficient section, however it is clear that this phenomenon does not exist for the stationary wheel but is a defining feature belonging to rotating isolated wheels. Fackrell [5] showed 
that it was the rotation of the wheel that moved the separation point upstream, and that the separation occurred not from the surface of the model, but in fact from the thin layer of fluid surrounding it that counteracted the freestream direction. Inaccurate prediction of separation can lead to incorrect prediction of the separation bubble and the flow that proceeds it, generating discrepancies with the experimental wake flow topology [17]. This dominant feature is therefore integral to accurate simulation, and visually this is shown in the majority of CFD results. It can be approximated that the point of separation is as follows; PIV Rotating Wheel $\left(265^{\circ}\right)$, CFD Rotating Wheel $\left(270^{\circ}\right)$ and CFD Rotating Wheel with MGP $\left(270^{\circ}\right)$.

Whilst the separation bubble is shown to be well represented for the rotating wheel, Figure 15, Figure 17 and Figure 18, the flow is seen to stay fully attached for the stationary wheel, Figure 14 and Figure 16 , with an increased $x$-velocity magnitude region than was numerically predicted, the high velocity flow structure extending slightly further in the PIV. Closer inspection of the vector direction in the PIV shows that the flow in this region and further downstream $\left(200^{\circ}<\theta<360^{\circ}\right)$ exhibits a strong downwash and remains attached until the rear geometry curvature and ground plane vortex interaction causes rear separation. This effect cannot be found behind a circular cylinder and is caused by the three-dimensional flow developed around the wheel. This was also found by Mears et al. [14] and computationally by Wäschle [16] and McManus and Zhang [22].

The in-plane, freestream normalized, velocity magnitude vector comparisons are shown in Figure 26 - Figure 30. For both PIV and CFD cases, the difference between the rotating and stationary wheels are shown to display similar contour trends with regards to the increasing or decreasing in-plane vectors. The difference in the freestream is marginally higher for the rotating PIV wheel, which was also found by Mears et al. [14], however the separation and therefore slower counter rotating flow is represented in both. The integral point here is that the difference between the two rotating wheel plots, Figure 28 and Figure 30, is marginal and close to zero. This is an example of where it may not be a necessity to include a moving ground plane to simulate the general global flow features of an isolated wheel accurately.

The cross plane results, located $5 \mathrm{~mm}$ behind the rear surface of the wheel, displayed in Figure 20 - Figure 22 are colored by the in-plane velocity magnitude using the $\mathrm{y}$ and $\mathrm{z}$ velocity vectors normalized by the freestream velocity, $\frac{U_{y, z}}{U_{\infty}}$. The set shown here only includes the numerical solution data. The moving ground plane only minimally affects the separation off the floor, as shown more clearly in difference plot Figure 30 as well as the 3D images, Figure 24 and Figure 25, which depicts the averaged, isosurface of $\mathrm{P}_{\mathrm{Tot}}=-100$ and mean wall shear stress (WSS) on the wheel and axle surface. The images displayed can be correlated with the revised wake model created by Knowles e al. [13], where the main difference between this and the model proposed by Cogotti [4] involves the elimination of the weak central pair of vortices created at the wheel sides. These are found to be swept up into the dominant top and bottom pair early on. This is also affected when a sting like structure is also used to support the wheel. The top pair, which are the weaker of the two in strength, are formed by the counter rotating flow off the wheel shoulders. The bottom two are created by the interaction with the floor boundary layer. The top pair is then found to merge with the bottom when further than a diameter away from the wheel downstream [16] and the development of this is shown in the 3D diagram, where the strong downwash over the top of the wheel feeds the upper vortex pair into the lower.

The noticeable difference evident between stationary and rotating CFD cross plane images and 3D diagrams, is due to the differing flow fields that exist aft of the model. For the stationary wheel, the flow over the crown stays attached for longer, accelerating and generating a higher velocity flow, clearly shown by the wall shear stress. This creates a larger front to back pressure difference for the stationary case which can be directly related to the increased drag. The rotation of the wheel creates an arch shaped vortex shown in Figure 24 and Figure 25 post crown and defined by characteristic $C$. This is a consequence of the stronger upper vortex pair A, sweeping more airflow from across the wheel sides into the near rear wake, the interaction of both causing the 'arch shape'. This phenomena is corroborated by both McManus \& Zhang [22] and Croner et al. [18], but is counter-argued by Wäschle [12] who found this vortex to be in the shape of a ring. Once again, minimal difference is evident between stationary and moving ground plane simulations The main variation between stationary and moving ground plane simulations is shown in the boundary layer produced as shown in Figure 30. The moving ground plane simulation does not form a boundary layer whilst the stationary ground plane has a boundary layer profile at the velocity inlet setup to match that of the Loughborough University Wind Tunnel. The effect of this is also shown in Figure 25 where the loss of the floor boundary layer eliminates the separation off the floor and the lower vortex right side structure is slightly larger. However, these images illustrate that once again there lies minimal difference between the stationary and moving ground plane simulations. This work leads to the conclusion that the changes made by the inclusion of a moving ground plane are smaller than those created by the rotation of the wheel. Thus it can be said that for research purposes, omitting a moving ground plane can be done if the global flow features are predicted with a good enough level of reliability with just the rotation of the wheel in place. 


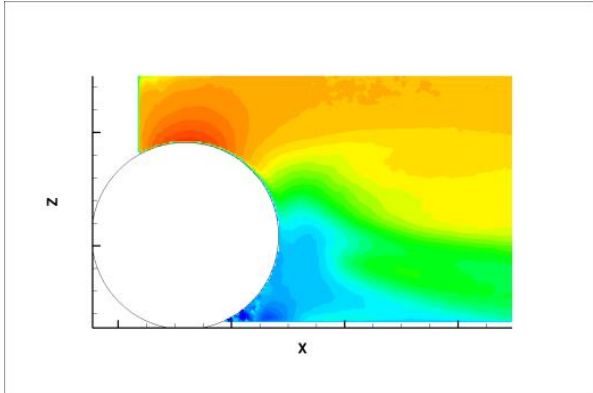

Figure 14. PIV Stationary Wheel: Normalised XVelocity

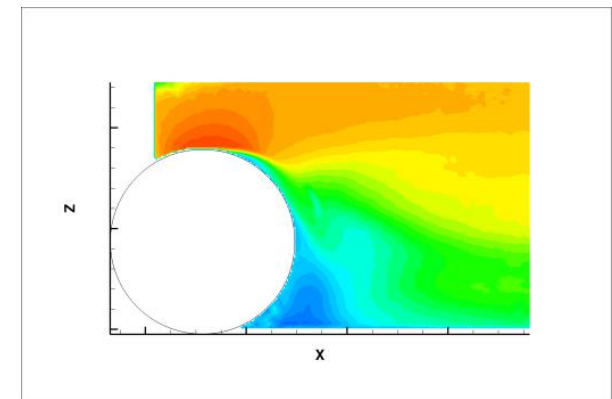

Figure 15. PIV Rotating Wheel: Normalised XVelocity

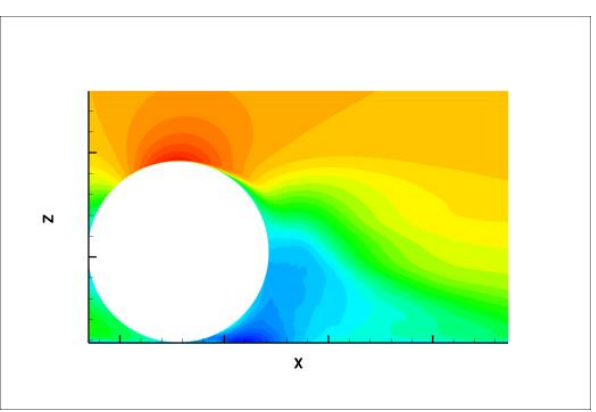

Figure 16. CFD Stationary Wheel: Normalised XVelocity

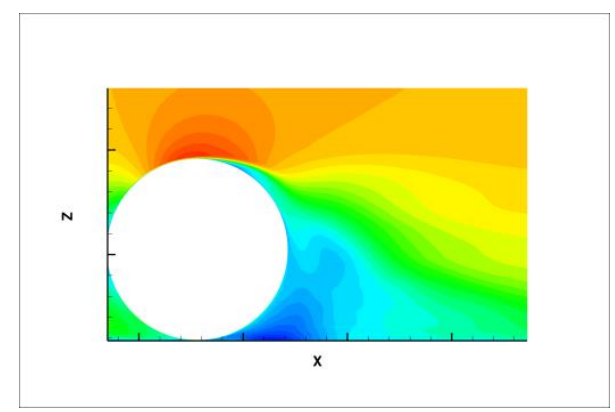

Figure 17. CFD Rotating Wheel: Normalised XVelocity

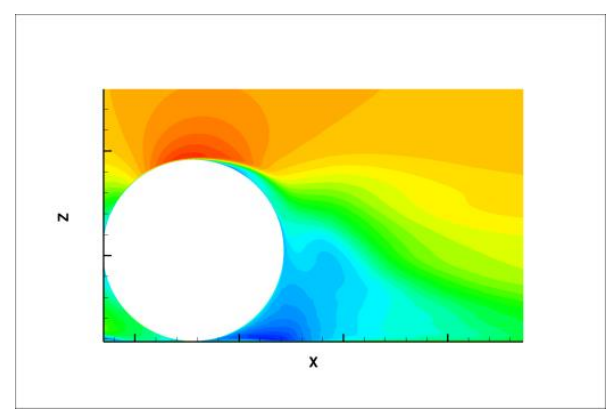

Figure 18. CFD Rotating MGP Wheel: Normalised X-Velocity

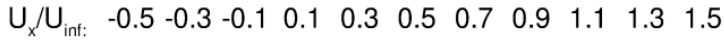

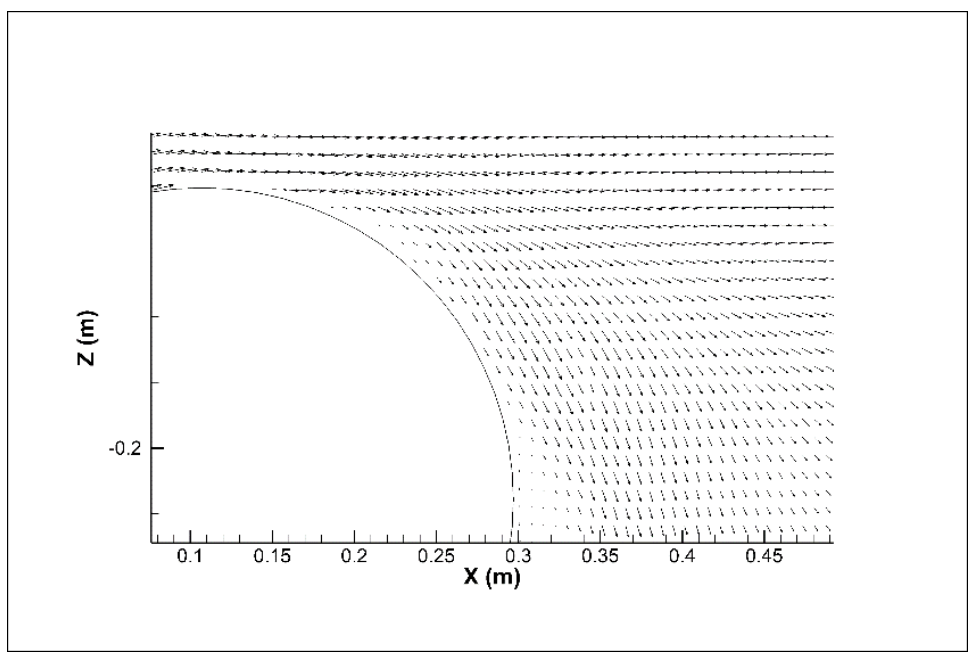

Figure 19. Stationary Wheel: PIV Velocity Vectors 


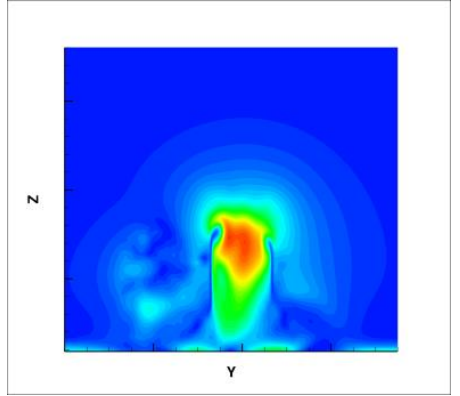

Figure 20. CFD Stationary Wheel: Cross Plane $\mathrm{U} / \mathrm{U}_{\infty}$

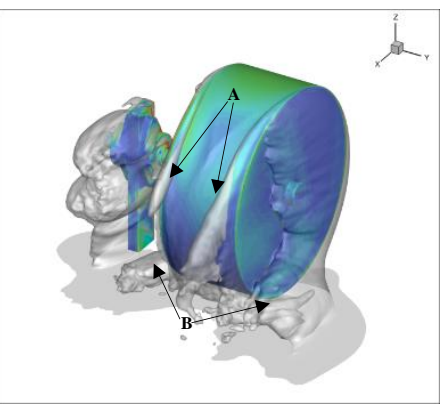

Figure 23. CFD Stationary Wheel: Total Pressure Isosurface and WSS

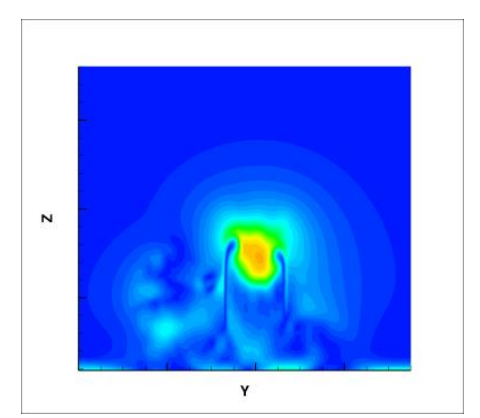

Figure 21. CFD Rotating Wheel: Cross Plane U/U
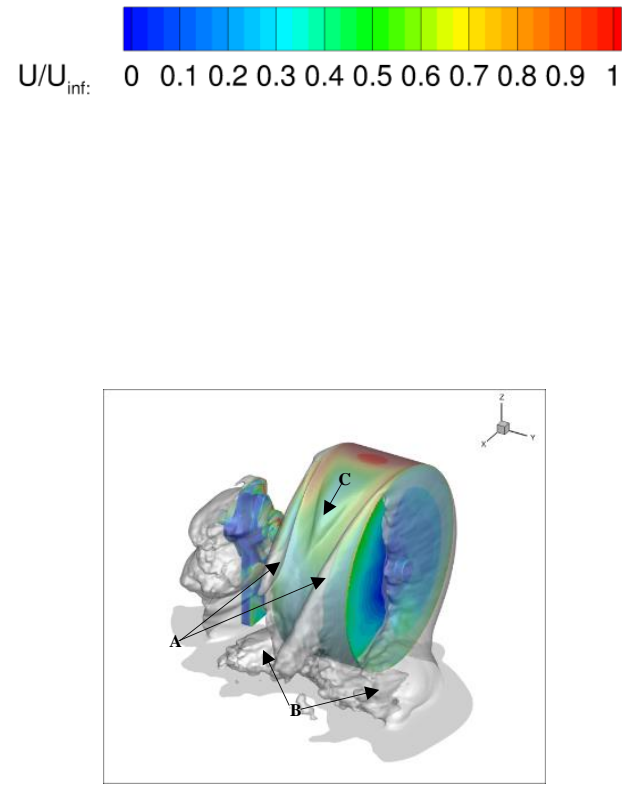

Figure 24. CFD Rotating Wheel: Total Pressure Isosurface and WSS

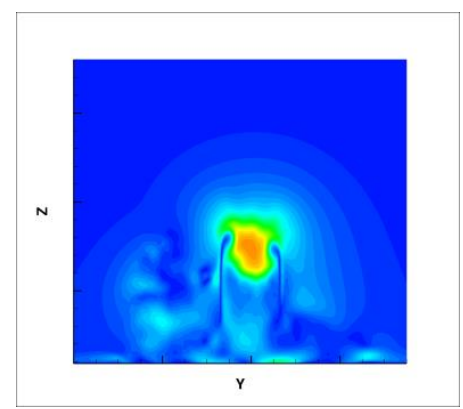

Figure 22. CFD Rotating MGP Wheel: Cross Plane $\mathrm{U} / \mathrm{U}_{\infty}$

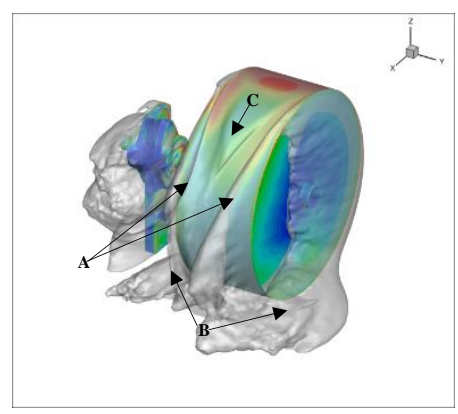

Figure 25. CFD Rotating MGP Wheel: Total Pressure Isosurface and WSS 


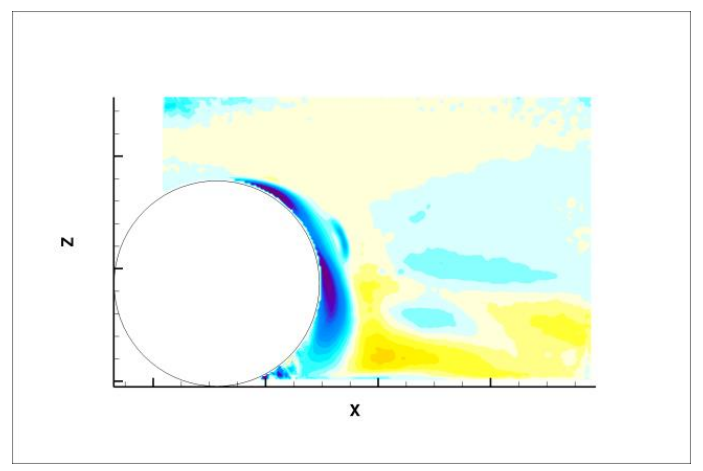

Figure 26. PIV U/U $\mathrm{U}_{\infty}$ Difference Rotating and Stationary

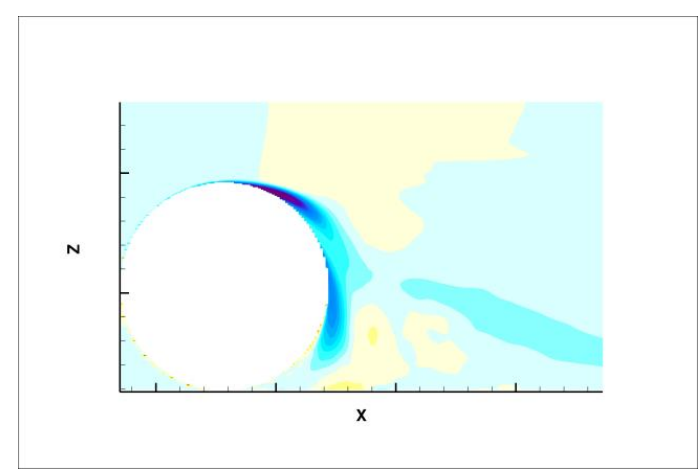

Figure 27. CFD U/U $\mathrm{U}_{\infty}$ Difference Rotating and Stationary

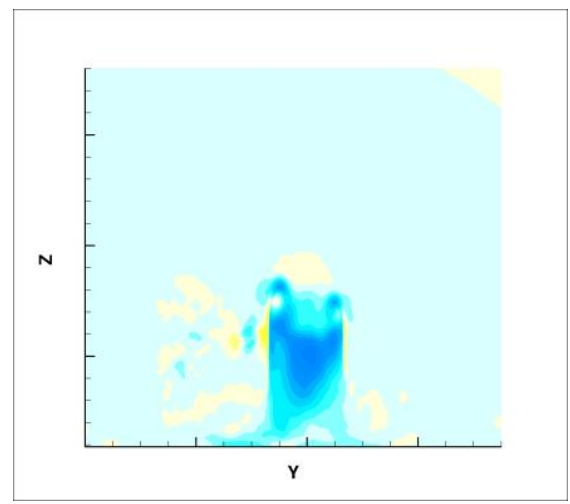

Figure 29. CFD Cross Plane U/U $\mathrm{U}_{\infty}$ Difference Rotating and Stationary

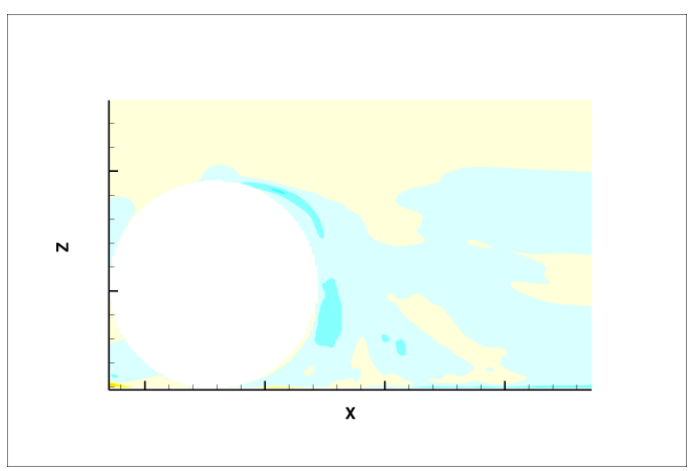

Figure 28. CFD U/U ${ }_{\infty}$ Difference Rotating w/o MGP

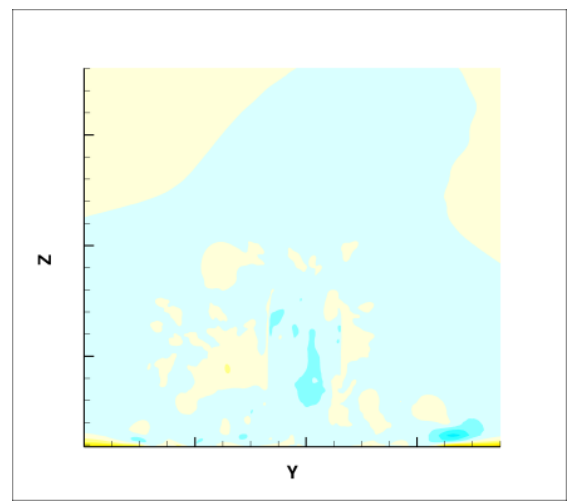

Figure 30. CFD Cross Plane U/U $\mathrm{U}_{\infty}$ Difference Rotating w/o MGP

Page 13 of 15 


\section{Conclusions}

- An isolated wheel rig was designed to investigate in depth the rotating wheel problem.

- The separation point and rear wake structure for the rotating wheel was well matched between the PIV and CFD giving confidence in the CFD.

- It was concluded that DES is the most computationally appropriate tool for analysis of flow around an isolated wheel, outperforming other models such as uRANS by capturing an increased amount of finer detailed, high frequency vortical structures.

- $\quad$ Stationary wheel balance and flow field PIV data matched with the numerical simulations.

- A comparison between the rotating wheel moving ground plane and stationary ground plane simulations was performed with trust in the previously validated computational data.

- Marginal difference was shown between these simulations, therefore it can be concluded that for research and design purposes, as generating the global flow field topology of a rotating wheel is not largely dependent on the movement of the ground plane but more so on the rotation of the wheel itself, it is reasonable to test without the use of a moving ground plane. However, for prototype validation, where there is a larger emphasis on accurate force measurements, a moving ground plane would still be required.

\section{References}

1. Hoerner, S.F., "Fluid-Dynamic Drag,” 2nd ed., Hoerner Fluid Dynamics, Bakersfield, California, 1965.

2. Morelli, A., "Aerodynamic Effects on a Automobile Wheel," 1969.

3. Stapleford, W.R. and Carr, G.W., "Aerodynamic Characteristics of Exposed Rotating Wheels," 1970.

4. Cogotti, A., "Aerodynamic Characteristics of Car Wheels," Int. J. Veh. Des. (Technical Advances in Vehicle Design Series, SP3, Impact of Aerodynamics on Vehicle Design):173-196, 1983.

5. $\quad$ Fackrell, J.E., "The Aerodynamics of an Isolated Wheel Rotating in Contact with the Ground," University of London, 1974.

6. Fackrell, J.E. and Harvey, J.K., "The Aerodynamics of an Isolated Road Wheel," in: Pershing, B., ed., Proceedings of the Second AIAA Symposium of Aerodynamics of SPorts and Competition Automobiles, Los Angeles, California, 1975.

7. Mercker, E., Breuer, N., Berneburg, H., and Emmelmann, H.J., "On the Aerodynamic Interference Due to the Rolling Wheels of Passenger Cars," SAE Trans. 100:460-476, 1991.

8. Mercker, E., Soja, H., and Wiedemann, J., "Experimental Investigation on the influence of Various Ground Simulation
Techniques on a Passenger Car.," Conference on Vehicle Aerodynamics, Royal Aeronautical Society, Loughborough, U.K., 1994.

9. Wickern, G., Zwicker, K., and Pfadenhauer, M., "Rotating Wheels - Their Impact on Wind Tunnel Test Techniques and on Vehicle Drag Results," SAE Technical Paper, SAE International, 1997, doi:10.4271/970133.

10. Elofsson, P. and Bannister, M., "Drag reduction mechanisms due to moving ground and wheel rotation in passenger cars," 2002 .

11. Lounsberry, T.H., Gleason, M.E., Kandasamy, S., Sbeih, K., Mann, R., and Duncan, B.D., "The Effects of Detailed Tire Geometry on Automobile Aerodynamics - a CFD Correlation Study in Static Conditions," SAE Int. J. Passeng. Cars - Mech. Syst. 2(1):849-860, 2009, doi:https://doi.org/10.4271/2009-01-0777.

12. Wäschle, a, "The Influence of Rotating Wheels on Vehicle Aerodynamics - Numerical and Experimental Investigations," SAE World Congr. 2007(724):776-790, 2007, doi:10.4271/2007-01-0107.

13. Knowles, R., Saddington, A., and Knowles, K., "SAE TECHNICAL On the Near Wake of Rotating, $40 \%$-Scale Champ Car Wheels," Mot. Eng. Conf. Exhib. (724), 2002.

14. Mears, A.P., Crossland, S.C., and Dominy, R.G., "An Investigation into the Flow-Field About an Exposed Racing Wheel," SAE World Congr. 2004(724), 2004, doi:10.4271/2004-01-0446.

15. Axon, L., Garry, K., and Howell, J., "An Evaluation of CFD for Modelling the Flow Around Stationary and Rotating Isolated Wheels," International Congress \& Exposition, SAE International, 1998, doi:https://doi.org/10.4271/980032.

16. Wäschle, A., Cyr, S., Kuthada, T., and Wiedemann, J., "Flow around an Isolated Wheel - Experimental and Numerical Comparison of Two CFD Codes," SAE World Congr. 2004(724), 2004, doi:10.4271/2004-01-0445.

17. Axerio-Cilies, J. and Iaccarino, G., "An Aerodynamic Investigation of an Isolated Rotating Formula 1 Wheel Assembly," J. Fluids Eng. 134:121101, 2012.

18. Croner, E., Bézard, H., Sicot, C., and Mothay, G., "Aerodynamic characterization of the wake of an isolated rolling wheel," Int. J. Heat Fluid Flow 43:233-243, 2013, doi:10.1016/j.ijheatfluidflow.2013.04.008.

19. Axon, L., Garry, K., and Howell, J., "The Influence of Ground Condition on the Flow Around a Wheel Located Within a Wheelhouse Cavity," Int. Congr. Expo. (724), 1999, doi:10.4271/1999-01-0806.

20. Regert, T. and Lajos, T., "Description of flow field in the wheelhouses of cars," Int. J. Heat Fluid Flow 28(4):616-

Page 14 of 15 
629, 2007, doi:10.1016/j.ijheatfluidflow.2007.04.017.

21. Pirozzoli, S., Orlandi, P., and Bernardini, M., "The fluid dynamics of rolling wheels at low Reynolds number," $J$. Fluid Mech. 706:496-533, 2012, doi:10.1017/jfm.2012.273.

22. McManus, J. and Zhang, X., "A computational study of the flow around an isolated wheel in contact with ground," ASME J. Eng. 128(May 2006):520-530, 2006, doi:10.1115/1.2175158.

23. Johl, G.S., "The Design and Performance of a $1.9 \mathrm{~m} \times 1.3 \mathrm{~m}$ Indraft Wind Tunnel," Loughborough University, 2010.

24. Newnham, P.S., "The Influence of Turbulence on the Aerodynamic Optimisation of Bluff Body Road Vehicles," Loughborough University, 2007.

25. Westerweel, J., "Fundamentals of digital particle image velocimetry," Meas. Sci. Technol. 8(12):1379, 1997.

26. CD-Adapco, "Star-CCM+: User Guide (Version 11.04)," https://stevedocs.cd-

adapco.com/starccmplus_latest_en/index.html?param=kTJw e, 2016.

Page 15 of 15 